\title{
Juventude, gênero e justiça reprodutiva: iniquidades em saúde no planejamento reprodutivo no Sistema Único de Saúde
}

\author{
Youth, gender and reproductive justice: health inequities \\ in family planning in Brazil's Unified Health System
}

Elaine Reis Brandão (http://orcid.org/0000-0002-3682-6985) ${ }^{1}$

Cristiane da Silva Cabral (http://orcid.org/0000-0003-3025-2404) ${ }^{2}$

${ }^{1}$ Instituto de Estudos em Saúde Coletiva, Universidade Federal do Rio de Janeiro. Praça Jorge Machado Moreira 100, Cidade Universitária. 21941598 Rio de Janeiro RJ Brasil.brandao@iesc.ufri.br

${ }^{2}$ Faculdade de Saúde Pública, Universidade de São Paulo. São Paulo SP Brasil.

\begin{abstract}
Sexual initiation is a gradual process of experimentation and learning the cultural repertoire of gender, reproduction, contraception, sexual violence and other topics surrounding youth sociability. Unlike sexual abstinence-based approaches promoted as a panacea for reducing "early pregnancy" in Brazil, reproductive justice is posited as a framework for addressing health inequities in family planning. This article discusses the challenges faced by public health policies in supporting adolescents and young people in their sexual and reproductive trajectories, drawing on the concept of intersectionality. We focus on public institutional initiatives providing long-acting reversible contraceptives (LARC) on the Brazilian Unified Health System (SUS) implemented over the last decade. We conducted a documentary anthropological study drawing on empirical data on contraceptive technologies in order to problematize what we call the "selective provision" of these devices and discriminatory and stigmatizing practices. Advocating the expansion of the provision of contraception on the SUS, with universal access to LARC for all women, distances itself from what we call "contraceptive coercion" among specific social groups.
\end{abstract}

Key words Youth, Long-acting reversible contraceptives, Intersectionality, Reproductive health
Resumo Iniciação sexual é um processo gradual de experimentação e aprendizado do repertório cultural sobre gênero, reprodução, contracepção, violência sexual, entre outros temas que circundam a sociabilidade juvenil. Ao contrário da perspectiva da abstinência sexual como panaceia para redução da "gravidez precoce" no Brasil, postula-se a abordagem da justiça reprodutiva como framework para enfrentar iniquidades em saúde no planejamento reprodutivo. Discutem-se desafios às politicas públicas de saúde para amparar adolescentes e jovens em suas trajetórias sexuais e reprodutivas, privilegiando a dimensão da interseccionalidade. O foco recai nas iniciativas institucionais públicas de oferta de métodos contraceptivos hormonais reversiveis de longa duração (LARC) no Sistema Único de Saúde, na última década. Do ponto de vista metodológico, tal reflexão se apoia em material empírico oriundo de pesquisa antropológica documental sobre tecnologias contraceptivas, no intuito de problematizar o que chamamos de "oferta seletiva" de tais dispositivose seu viés discriminatório e estigmatizante. A defesa da ampliação da oferta contraceptiva no SUS, com acesso universal de todas as mulheres à LARC, distancia-se do que designamos como "coerção contraceptiva" em determinados grupos sociais.

Palavras-chave Juventude, Contracepção reversivel de longo prazo, Interseccionalidade, Saúde reprodutiva 


\section{Introdução}

A iniciação sexual é um processo gradual de experimentação e de aprendizado do repertório cultural sobre gênero, reprodução, contracepção, violência sexual, entre outros temas que circundam a sociabilidade juvenil. Ao contrário da perspectiva da abstinência sexual como panaceia para redução da "gravidez precoce" no Brasil', postulase a abordagem da justiça reprodutiva como framework para enfrentar as iniquidades em saúde no tocante ao planejamento reprodutivo.

$\mathrm{O}$ tema da gravidez na adolescência voltou à cena com vigor em recente debate público no Brasil ${ }^{2,3}$. A maioria dos analistas concorda que a perspectiva teórica e sociopolítica de garantia e afirmação dos direitos sexuais e reprodutivos aos adolescentes e jovens, assegurados ao longo de décadas de ativismo social feminista e LGBT+, árduo trabalho diplomático nas Conferências das Nações Unidas, e na formulação de políticas públicas de saúde ao segmento juvenil ${ }^{4,5}$ deve prevalecer como parâmetro de respeito aos direitos humanos.

No entanto, uma inflexão importante tem surgido nas últimas décadas, na direção de problematizar obstáculos estruturais a tais garantias sobretudo às mulheres pobres e negras, em contextos de extrema desigualdade social ${ }^{6}$. A dimensão de advocacy, pautada em preceitos legais no tocante aos direitos sexuais e reprodutivos é fundamental, garantindo a autonomia do sujeito (homens e mulheres) em suas escolhas sexuais e reprodutivas. Mas a vinculação deste pressuposto ao paradigma liberal, individualista, obscurece o racismo estrutural existente nas sociedades ditas democráticas, o qual impõe obstáculos muitas vezes intransponíveis para o direito de escolha e de acesso aos insumos biomédicos e serviços de saúde a determinados grupos populacionais. Em diálogo com o paradigma da interseccionalida$\mathrm{de}^{7,8}$ como cruzamento de diversas hierarquias sociais - classe, raça, gênero, sexualidade, etnia, idade, nacionalidade - que se potencializam e produzem novas e complexas discriminações, a perspectiva da justiça reprodutiva reivindica a garantia da justiça social a grupos de mulheres tradicionalmente excluídos. Isso inclui garantia por parte do Estado de outros direitos sociais básicos, como educação, moradia, trabalho, alimentação, dentre outras dimensões fundamentais à manutenção da vida humana em condições dignas. A abordagem da justiça reprodutiva busca ampliar a luta pelo direito ao aborto - central ao movimento pro-choice norte-americano - para incluir também o direito a ter um filho, e a ter um filho em condições dignas, no caso de mulheres encarceradas ou em situação de rua, por exemplo ${ }^{9,10}$.

O tema da reprodução entre jovens negras e pobres está relacionado com suas expressivas dificuldades para acessar serviços de saúde, (im) possibilidade de parir em condições dignas ${ }^{11}$, ao sofrimento e maus-tratos no atendimento pós-aborto ${ }^{12}$, além de uma miríade de procedimentos legais, jurídicos, socioassistenciais que, a despeito de proteger seus interesses, negam sua liberdade sexual e reprodutiva, chegando a criminalizar as práticas reprodutivas neste segmento populacional, intervindo em seus corpos de forma coercitiva. São tecnologias de governos que tutelam seus corpos, punem suas decisões reprodutivas, confiscam seus filhos, entre outras ações de caráter arbitrário ${ }^{13-15}$.

Essa discussão não é nova. Respeitadas intelectuais feministas e ativistas negras ${ }^{16,17}$, já produziram nesta direção, nos sensibilizando e alertando sobre a necessidade de identificação do racismo institucional como motor de algumas políticas públicas.

Buscamos associar em nossa perspectiva de análise sobre o planejamento reprodutivo no Brasil, a partir da compreensão da justiça reprodutiva, como a expansão do processo de biomedicalização em sociedades excludentes e altamente desiguais ocorre de forma socialmente estratificada, generificada e racializada ${ }^{18-20}$. Para tanto, tomamos os métodos contraceptivos hormonais reversíveis de longa duração (LARC) como objeto de estudo para refletir sobre a necessária ampliação da oferta contraceptiva no Sistema Único de Saúde (SUS) e o modo como ela vem sendo incrementada.

$\mathrm{O}$ artigo discute os desafios às políticas públicas de saúde para amparar adolescentes e jovens em suas trajetórias sexuais e reprodutivas, privilegiando o exame das iniciativas institucionais públicas de oferta de LARC no SUS, na última década. Busca-se problematizar o que chamamos de "oferta seletiva" de tais dispositivos e seu viés discriminatório e estigmatizante, que designamos como "coerção contraceptiva" em determinados grupos sociais.

\section{Justiça reprodutiva e LARC}

O uso de tecnologias biomédicas (contraceptivas ou não) em estudos clínicos com populações consideradas "vulneráveis" não é um fato novo no país $\mathrm{s}^{21,22}$. E quase sempre o apelo de profissionais de saúde, gestores públicos e re- 
presentantes de laboratórios farmacêuticos para a promoção de iniciativas que busquem mediar o acesso a novas tecnologias biomédicas (caras em geral para ampla utilização) a determinados grupos populacionais prioriza a perspectiva dos direitos sexuais e reprodutivos, capturando o discurso politicamente hegemônico que visa garantir direitos fundamentais a jovens mulheres. O consumo de determinados dispositivos biomédicos funcionaria como passaporte à cidadania e modernidade, sem aporte de políticas sociais para transformação de suas condições de vida.

Embora os LARC sejam recomendados por associações médicas e organismos internacionais ${ }^{23,24}$ como altamente eficazes, sua ampla utilização não deve ser tomada como única forma de enfrentamento das iniquidades em saúde reprodutiva. Em editorial recente na revista American Journal of Public Health ${ }^{25}$, notórias pesquisadoras do campo de estudos sociais da ciência, do gênero e da reprodução problematizam as recomendações da American Academy of Pediatrics, que considera os LARC “first-line contraceptive choices" para adolescentes e jovens adultas. As autoras criticam a redução da abordagem da gravidez na adolescência ao comportamento individual feminino, perpetuando-se injustiças estruturais. Solicitam cautela na recomendação de LARC a grupos adolescentes, alertando para os vieses de raça e classe que tais aparentes soluções ensejam, ao ferir a autonomia reprodutiva de suas usuárias. Advogam "an approach to LARC informed by reproductive justice and predicated on the equal value of all lives" 25 (p.18).

\section{Coerção contraceptiva: categoria sociológica fundamental para exame das políticas públicas}

Buscando desvendar a genealogia do termo "coerção contraceptiva” em programas de planejamento familiar, na era pós Cairo, Senderowicz ${ }^{26}$ evidencia a sofisticação que tal categoria pode adquirir distanciando-se de uma compreensão simplista que a equipara a um ato violento de alguém contra outra pessoa. Amparada no paradigma da justiça reprodutiva, a autora sinaliza para uma dimensão oculta que integra processos estruturais relativos aos sistemas de saúde e agentes do Estado (como quotas, metas, indicadores, custos, cálculos e estimativas, etc.), mediados por intervenções individuais, aparentemente neutras, técnicas.

A autora destaca a dupla direcionalidade que a coerção contraceptiva pode adquirir, desde a força para aceitação de determinado método contraceptivo que a mulher não quer, até ser impedida ou ter dificuldades para acessar métodos que elas desejam, comumente conhecidas como "barreiras de acesso" ou "provider bias". Estas são frequentemente mais aparentes no tocante às estigmatizações sofridas por adolescentes e jovens, não casadas/unidas, em relação ao exercício de sua sexualidade.

Senderowicz ${ }^{26}$ nos adverte para uma gradação de práticas que comumente não costumamos tomá-las como coercitivas, mas que obstaculizam a autonomia reprodutiva feminina, tal como uma oferta restrita de métodos contraceptivos que não contemple as necessidades das mulheres em diferentes contextos relacionais, socioculturais, geracionais. Há um espectro na abordagem à coerção, desde a dimensão estrutural até a interpessoal: informações médicas parciais (vantagens do método e não seus efeitos colaterais), aconselhamentos enviesados ou dirigidos, limitados mix de métodos, insistências até que as mulheres aceitem tal ou qual método, ameaças de restrição na atenção à saúde futura, inserção de métodos sem o conhecimento ou consentimento, em especial no período pós-parto, recusa de remoção de LARC sob pedido da usuária, entre outros exemplos ${ }^{26}$.

Ao refletir sobre as estratégias institucionais que presidem o planejamento reprodutivo no país e as nuances que o conceito de "coerção contraceptiva" permite vislumbrar, indaga-se de que modo as iniquidades em saúde, as desigualdades de classe, raça, gênero são consideradas para uma oferta ampliada de métodos contraceptivos no SUS, que respeite as especificidades e necessidades juvenis em contextos de extrema pobreza e (in)justiça reprodutiva. Nosso argumento se apoia na observação da restrição das possibilidades de escolha dentre diversas alternativas de métodos contraceptivos no SUS, incluindo o acesso à laqueadura tubária, e uma oferta dirigida de LARC a certos perfis femininos, contrariando a universalidade da oferta contraceptiva indistintamente às mulheres em idade reprodutiva.

\section{Método}

Trata-se de pesquisa antropológica, de natureza documental, em fontes de acesso público disponíveis no ciberespaço, a qual integra o projeto "Direitos sexuais e reprodutivos em debate: desvendando sentidos e usos sociais de dispositivos biomédicos para contracepção e esterilização em 
mulheres", em diálogo com o projeto "Juventudes, sexualidade e reprodução: um estudo sobre mudanças e permanências nas trajetórias sexuais e reprodutivas de jovens brasileiros no cenário das relações sociais mediadas pelas redes sociais", coordenados pelas autoras. Nesta primeira fase da pesquisa, procedeu-se à observação das notícias veiculadas na mídia eletrônica a respeito dos dispositivos LARC e coleta de material empírico, como documentos legais e institucionais (projetos de lei, decretos, protocolos, etc.), que informam sobre disponibilidade de tais dispositivos na rede pública de saúde do país, trabalhos acadêmicos, position papers emitidos pela sociedade civil organizada, dentre outros.

Os LARC ainda não estão disponíveis na rede pública de saúde no Brasil, de forma ampla e universal a todas as mulheres. Estamos nos referindo basicamente ao implante subdérmico, liberador de etonogestrel (conhecido como Implanon ${ }^{\circledR}$ ), com duração de três anos, e o sistema intrauterino com levonorgestrel (conhecido como DIU Mirena ${ }^{\circledR}$ ), com duração para cinco anos. $\mathrm{O}$ artigo se detém na análise desta documentação, com enfoque especial no público eleito para receber tais dispositivos, no âmbito das iniciativas, sempre designado pelas categorias "adolescentes", "mulheres em vulnerabilidade", "mulheres em risco", no intuito de configurar o que chamamos de "oferta seletiva" destes métodos contraceptivos no SUS. Nossa intenção não é examinar a operacionalização de cada iniciativa, mas apreender como os gestores públicos as anunciam, ou seja, sob que linhas centrais elas são comunicadas ao conjunto da sociedade como medidas benéficas às mulheres.

Tais categorias nativas que emergem do material empírico nos inspiram a refletir sobre uma classificação social prévia, subjacente às iniciativas, que atribui de modo simplista os altos índices de morbimortalidade materna, de gravidez na adolescência ou imprevista, de infecções sexualmente transmissíveis, até mesmo de condição de pobreza à incapacidade de certas mulheres se cuidarem, evitando uma gestação. Frente a tal indisciplina feminina, lançam mão de tecnologias de governo, no intuito de transferirem tal responsabilidade ao Estado e seu aparato biomédico.

Adotamos o conceito de "coerção contraceptiva"26 para analisar esta sujeição identificada na publicização destas iniciativas compiladas, que acomete sistematicamente mulheres jovens, negras, pobres, em situação de exclusão social, embora tais iniciativas sejam sempre anunciadas com entusiasmo como promotoras de "cuidado" e "proteção" às usuárias.

\section{Resultados \\ Iniciativas institucionais de oferta de LARC no SUS}

Seguidamente temos assistido no Brasil, por meio de divulgação na mídia, a iniciativas institucionais de oferta dos LARC em serviços de saúde que integram o SUS. São iniciativas esparsas, no tempo e no espaço da federação, gestadas em sua maioria em âmbito municipal ou estadual, mas que revelam um traço peculiar: são destinadas a certo grupo específico de mulheres, consideradas "aptas" a utilizar tal método contraceptivo. Em geral, são adolescentes, jovens em situação de rua, em uso ou dependentes de substâncias químicas, em contexto prisional ou asilar, privadas de liberdade, portadoras de HIV.

Três iniciativas de oferta de LARC no sistema público de saúde exemplificam nosso argumento: o estado do Ceará, no nordeste brasileiro, que desde 2010, vem promovendo a inserção destes métodos na capital e cidades do interior; o município de São Paulo, que mais recentemente (2018) aprovou projeto de lei a respeito, e o estado do Rio Grande do Sul, que protagonizou um episódio de repercussão nacional, em 2018, envolvendo adolescentes em acolhimento institucional.

A notícia em 22 de setembro de 2010 no Diário do Nordeste traz em destaque: "CE é o $1^{\circ}$ do Brasil a usar implante contraceptivo"27. A reportagem traz, em tom entusiástico, a oferta do implante subdérmico no programa de "planejamento familiar especial" da Secretaria de Saúde do Estado (Sesa), destacando que "ele" permite evitar "a gravidez indesejada e, principalmente, o óbito materno". O texto informa que "inicialmente, o medicamento está sendo utilizado em mulheres acompanhadas no Hospital Geral Dr. César Cals, unidade da rede estadual referência em assistência à gravidez de risco", mas gradativamente seria distribuído nas Coordenadorias Regionais de Saúde no interior.

Embora o planejamento reprodutivo sempre se apresente com uma justificativa consensual, difícil de ser contestada, como a meta de reduzir a mortalidade materna, o exame da declaração pública do gestor explicita outra dimensão da intencionalidade da medida:

Segundo o médico obstetra e responsável pela Saúde da Mulher na Sesa [...], a aplicação dos 
medicamentos será importante nas adolescentes. Pois elas, por muitas vezes, esquecem-se de tomar os anticoncepcionais diários e são as que mais engravidam de forma indesejada. "O Implanon oferecido pela Sesa é destinado, principalmente, às adolescentes, mulheres com doenças sexualmente transmissiveis, dependentes químicas e quem está cumprindo pena na prisão"27.

Em julho de 2016, duas outras reportagens destacam a oferta do implante contraceptivo no Ceará a "mulheres em situação de risco social"28,29. Em uma delas, a então supervisora do Núcleo da Saúde da Mulher apoia-se em argumentos como esquecimento de ingestão da pílula anticoncepcional ou não acompanhamento correto por parte das adolescentes, o que incorre, portanto, na "realização de abortos clandestinos ou, ainda, o abandono dos estudos para cuidar do bebê". E ressalta: "Além disso, este é um dos melhores métodos para elas por ser contínuo, porque as adolescentes não usam camisinha" ${ }^{28}$.

O projeto no Ceará tem parceria com a Secretaria da Justiça do Estado e a Secretaria Especial de Políticas sobre Drogas, e apoio da Sociedade Cearense de Obstetrícia e Ginecologia para atender "mulheres em situação de vulnerabilidade". O programa se destina a adolescentes de 15 a 19 anos, mulheres em idade fértil que estão em situação de privação de liberdade e mulheres em situação de dependência química.

O portal do Governo do Estado do Ceará apresenta a defesa do uso de LARC para "populações vulneráveis" ou com dificuldade de acesso ao sistema de saúde, sobretudo no pós-parto, o que asseguraria uma "redução de gestações não planejadas e um intervalo intergestacional seguro (maior que 18 meses)", tais como em "adolescentes e usuárias de álcool e outras drogas", que deixariam "a maternidade com seu planejamento familiar já resolvido"29.

Passemos ao sudeste do país, no município de São Paulo, onde desde 2015 tramitava o projeto de Lei 467/2015 sobre o tema, de autoria da vereadora P. Bezerra (PSDB), aprovado em dezembro de 2017, na Câmara Municipa ${ }^{30}$. Em 19 de janeiro de 2018, o prefeito de São Paulo sancionou a Lei no 16.806 que "Dispõe sobre política de proteção às mulheres em situação de vulnerabilidade pela Rede Pública de Saúde, com a utilização do Contraceptivo Reversível de Longa Duração de Etonogestrel, e dá outras providências" (grifo nosso $)^{31}$. As justificativas públicas para tal iniciativa são baseadas em indicadores de saúde, como redução do número de gestações não planejadas, e das taxas de mortalidade infantil e materna. "Dependentes químicas, moradoras de rua e adolescentes terão prioridade na distribuição gratuita, tanto na rede pública convencional, quanto por meio de instituições conveniadas" ${ }^{32}$.

Em fevereiro de 2016, após projeto piloto em um hospital maternidade municipal, a Secretaria Municipal de Saúde de São Paulo anuncia a compra de 1.000 implantes contraceptivos para ampliar a distribuição na rede de saúde, sinalizando para a economia que tais dispositivos poderiam acarretar.

No texto divulgado pela SMS-SP, reaparece um argumento recorrente para a recomendação deste método ${ }^{33}$ : o fato de serem de longa ação, oferecerem proteção contra a gravidez em longo prazo (três anos) e "não exige disciplina da mulher, pois não é de uso diário". Embora destaquem que tais contraceptivos possam "ser interrompidos a qualquer momento, caso haja a vontade de engravidar, recuperando a fertilidade preexistente de forma rápida logo após a remoção", não se explicita que a remoção é médico-dependente, ou seja, a usuária precisará encontrar algum médico que o faça sem cobrar tal procedimento.

Pouco mais de um ano depois da Lei Municipal no 16.806 (janeiro de 2018), o Decreto $\mathrm{n}^{\circ}$ 58.693, de 2 de abril de $2019^{34}$, vem regulamentá -la, dispondo no art. $1^{\circ}$ :

As mulheres da Cidade de São Paulo em situação de vulnerabilidade, atendidas na Rede Pública de Saúde, por meio de unidades diretas ou entidades conveniadas a qualquer título, terão direito à inserção gratuita de implantes contraceptivos reversiveis de longa duração de etonogestrel, conforme protocolo da Secretaria Municipal da Saúde, respeitada a sua livre opção ${ }^{34}$ (grifo nosso).

$\mathrm{O}$ artigo $2^{\circ}$ especifica quem são as "mulheres em situação de vulnerabilidade": dependentes químicas; mulheres em situação de rua; e "adolescentes de regiões com vulnerabilidade social muito alta, conforme definido pelo Índice Paulista de Vulnerabilidade Social - IPVS 2010, elaborado pela Fundação SEADE”. Por fim, o art. $3^{\circ}$ ao dispor que "As usuárias deverão ser informadas, pelos profissionais capacitados das Unidades de Saúde de Referência do Sistema Municipal de Saúde, sobre os benefícios, riscos, efeitos colaterais e duração deste método contraceptivo", parece vir a corrigir eventuais desvios ou interpretações vagas que a lei municipal deixava em aberto. Originalmente, o projeto de lei de 2015 continha um paragrafo único com lista exaustiva de categorias de mulheres consideradas "em situação de vulnerabilidade social" (profissionais do sexo, portadoras de HIV, adolescentes meno- 
res de 17 anos com gestações anteriores ou com "baixa adesão os serviços de saúde", etc.), o qual foi vetado pelo Prefeito ao sancionar a lei.

Nosso último exemplo está situado na cidade de Porto Alegre-RS, onde um episódio recente despertou celeuma entre representantes da sociedade civil organizada, feministas, Conselho Municipal de Saúde, dentre outras vozes que integram a formulação de políticas públicas de saúde. O Ministério Público do Estado divulgou a celebração de um “Termo de Cooperação" com o Município de Porto Alegre (Secretaria Municipal de Saúde $)^{35,36}$, dois hospitais públicos, um deles universitário, e a empresa farmacêutica Bayer para a inserção de 100 dispositivos intrauterinos (SIU-LNG), com ação anticoncepcional por 5 anos, nas adolescentes em situação de acolhimento institucional sob tutela do Estado.

Uma série de protestos e críticas rapidamente foram veiculados, sinalizando que tal "Termo de cooperação" havia passado ao largo das instâncias de controle social que debatem questões atinentes à saúde da população ${ }^{37,38}$. O termo tinha vigência de 2 anos, previa uma consulta de revisão até 45 dias após a inserção do dispositivo, mas não explicitava como seria o acompanhamento clínico às adolescentes, em uso de método hormonal ao longo de cinco anos (vigência do produto), nem como seria garantido sua retirada, mesmo após decorrido este tempo, tendo em vista que a permanência no abrigo finda ao se completar 18 anos, extinguindo-se assim o vínculo com a instituição. Em abril de 2019, o Tribunal Regional Federal do RS suspendeu tal "Termo de cooperação" ${ }^{39}$.

Este evento trouxe à tona iniciativa anterior (2006), no município de Porto Alegre, de outro programa com aval da Secretaria Municipal de Saúde para inserção de 2.500 implantes contraceptivos em adolescentes de 15 a 18 anos em dez bairros da periferia da $\operatorname{cidade}^{40}$. Na ocasião, autoridades, grupos organizados feministas e de defesa dos direitos de crianças e adolescentes se mobilizaram para discutir e questionar a legitimidade de tais ações públicas que feriam direitos das adolescentes ${ }^{41}$. Tal iniciativa também ocorreu à revelia do debate público sobre politicas de assistência à saúde de adolescentes no município, tendo sido suspensa no ano seguinte por ordem judicial.

A categoria "mulheres em situação de vulnerabilidade social" tende a ser uma "chave mágica" ou um "passe-livre" para justificar ações de planejamento reprodutivo sob lógica do Estado, em particular voltadas para adolescentes e jovens negras. Certamente, sua qualificação está em disputa, servindo a diversos interesses políticos e pode ser subjetivamente interpretada. Por exemplo, por que nunca se mencionam mulheres que sofrem violência doméstica, de seus parceiros, como destinatárias destes programas? Elas não estariam vivendo em situação de risco social? Por que a reprodução nas franjas da marginalidade incomoda tanto? $\mathrm{O}$ fato de existirem sujeitos que vivem toda uma existência excluídos socialmente não deveria gerar maior indignação por parte do Estado?

\section{Discussão}

\section{“Oferta seletiva” de LARC: entre ampliação e violação de direitos}

Embora as iniciativas de oferta de LARC se amparem em indicadores de saúde, a face coercitiva nos parece evidente: são ações dirigidas a grupos específicos de mulheres (adolescentes, em situação de rua, dependentes químicas), que certamente se encontram em condições precárias de vida e frágeis para fazer face ao poder médico/ estatal, caso não desejem a inserção de LARC.

Essa face coercitiva vem travestida de termos como "proteção à saúde" das mulheres em situação de vulnerabilidade social. Não se menciona nos documentos analisados qualificação de corpo técnico-profissional para abordagem da educação em sexualidade que inclua as questões de gênero e violência sexual. Há apenas treinamento técnico para inserção dos dispositivos. $\mathrm{O}$ foco parece ser economizar recursos com "maternidade de risco"13, via LARC. Há um pressuposto de uma necessidade maior para a intervenção e instrumentalização destes corpos, para reduzir danos maiores relativos à reprodução entre pobres e negros.

Em trabalho anterior, discute-se a solicitação feita pela Federação Brasileira das Associações de Ginecologia e Obstetrícia (FEBRASGO) ao Ministério da Saúde, no ano de 2015, para incorporação destas tecnologias contraceptivas no SUS com indicações para uso em "populações especiais" (mulheres jovens de 15 a 19 anos) ${ }^{42}$, negada naquela ocasião. Ao analisar tal pleito, estava subjacente à avaliação dos especialistas a incapacidade das jovens controlarem sua fecundidade, se cuidarem, sendo portanto mais eficaz transferir tal responsabilidade ao médico, de forma razoavelmente duradoura ( 3 ou 5 anos), por meio da inserção destes métodos. 
A análise das iniciativas apresentadas delineia um cenário em que as adolescentes e jovens ficam à mercê do discernimento dos profissionais de saúde, com seus valores e juízos morais, preconceitos raciais e classistas, sobre tais mulheres e seu comportamento sexual e reprodutivo "desviante”. Portanto, é preciso explicitar o que está oculto em tais iniciativas: algumas "maternidades são possíveis", outras não.

Assim, oscilamos no Brasil entre suprimir o sexo na adolescência, vide debate público antes citado, ou propor medidas de contenção à gravidez na adolescência revestidas de ações de "proteção à adolescência vulnerável”, em que a oferta seletiva de LARC é um caminho pragmático. Sabemos que a simples inclusão de um Termo de Consentimento Livre e Esclarecido (da adolescente ou jovem mulher e/ou responsável) para garantir “adesão voluntária” à LARC necessita ser problematizada, tendo em vista a dificuldade de sujeitos sem educação científica para compreender termos técnicos, os modos de convencimento existentes que permeiam a autoridade de profissionais de saúde, em relação hierárquica com tais usuárias, dentre outras questões éticas importantes. Esse debate ético também nos parece bastante silenciado no país.

Impossível não adicionar a esse contexto o debate demográfico sobre juvenilização da reprodução no Brasil, bem como suas implicações nas duas últimas décadas a partir da Lei de Planejamento Familiar ${ }^{43}$. A transição demográfica no Brasil não implicou uma postergação da reprodução para faixas etárias mais elevadas, mantendo-se um perfil de fecundidade jovem (faixas etárias de 20-24 e 15-19) (4 $^{4}$ Junto a isso, mantém-se a querela do alto percentual de gestações imprevistas, bem como a da reprodução em momento inoportuno. A regulamentação da esterilização voluntária com a Lei de Planejamento Familiar estabeleceu critérios para realização cirúrgica em território nacional (ter ao menos 25 anos de idade ou dois filhos vivos), mas essa ação veio acompanhada de alguns desdobramentos inesperados.

Por um lado, ela foi saudada como importante avanço em termos da possibilidade de democratização do acesso para o procedimento em relação ao que vinha sendo praticado até então (ampliação da oferta à população masculina, refreio à "epidemia de laqueaduras" entre as mulheres). Todavia, a observação da diminuição do número de esterilizações femininas gerou questionamentos sobre se as condições estipuladas para realização da laqueadura tubária teriam acabado por promover novas barreiras de acesso das mulheres, sobretudo das mais jovens. Infelizmente, o Brasil enfrenta longo período de ausência de informações populacionais no que concerne ao comportamento contraceptivo, pois a última pesquisa sobre demografia e saúde (PNDS) data de 2006.

Pode-se especular sobre um certo vácuo deixado no campo das ofertas contraceptivas a partir dessa dinâmica inesperada em relação à esterilização feminina, o qual poderia estar sendo paulatinamente ocupado pela "oferta seletiva" de LARC. Desfecho frequente entre mulheres que começam a trajetória reprodutiva cedo, a esterilização costuma ser um método contraceptivo atraente, sobretudo entre as que alcançam rapidamente o "número de filhos desejados", e foi inúmeras vezes colocada enquanto solução para encerrar as trajetórias reprodutivas de "mulheres abjetas". Os LARC representam, neste momento, "método ideal" para o adiamento (e espaçamento) da reprodução, mas podem também se constituir em potenciais artefatos para encerramento da trajetória reprodutiva.

Percebidos como em estado de descontrole latente e, portanto, em permanente estado potencial de reprodução, os corpos femininos "carecem” de intervenção. Assim, tais questões nos levam novamente ao debate sobre um certo "malthusianismo social", não apenas dos profissionais de saúde, mas de determinadas ações e políticas públicas postas em curso "em nome" da saúde das mulheres e/ou dos seus direitos reproduti$\operatorname{vos}^{45}$. À histórica oferta seletiva da laqueadura tubária vem se somar a proposição coercitiva dos LARC a determinados segmentos populacionais femininos. Há similaridades nestes processos que nos impelem a pensar sobre estratégias em curso para governamentalidade dos corpos.

\section{Considerações finais}

A atenção dos serviços públicos de saúde no tocante a prover cuidados relativos à sexualidade, ao planejamento e à saúde reprodutiva de jovens deve resguardar princípios éticos de respeito à integridade e à autonomia pessoal, sem qualquer ordem de constrangimentos por parte de profissionais de saúde. O planejamento reprodutivo como política pública de saúde, com foco especial na adolescência e juventude, não se faz prescindindo do debate sobre gênero, sexualidade, racismo, violência de gênero nas escolas de ensino fundamental e médio, na formação universitária, na mídia, tampouco de ampla distribuição de 
preservativos em locais de sociabilidade juvenil, da provisão de outros métodos contraceptivos (pílula oral, contracepção de emergência, injetáveis, DIU de cobre), do acesso ao aborto seguro, do acesso à laqueadura tubária ou vasectomia, livre de aprovação do cônjuge, apoiando tais jovens em suas decisões.

Ao tomar os LARC como objeto de estudo para refletir sobre o planejamento reprodutivo no SUS, fica patente que, ao contrário de outros momentos históricos, nos quais a formulação de políticas públicas de assistência integral à saúde de mulheres e jovens incluía ampla participação social de representantes destas usuárias, para discussão de seus interesses, atualmente os interlocutores privilegiados são as associações médicas, os gestores públicos, bem como os laboratórios farmacêuticos interessados. Não foi ao acaso que a organização não-governamental SisterSong. Women of Color Reproductive Justice Collective, berço do debate sobre justiça reprodutiva nos EUA, lançou em 2016 um manifesto em conjunto com National Women's Health Network, assinado por múltiplas instituições de defesa dos direitos das mulheres, intitulado LARC - Statement of Principles ${ }^{46}$, reassegurando a liberdade das mulheres e as necessárias condições para que a escolha de um método contraceptivo seja feito "de maneira medicamente ética e culturalmente competente".

Por fim, o conceito de "coerção contraceptiva" nos possibilitou iluminar o quanto a oferta de LARC dirigida a grupos populacionais específicos reifica um modus operandi de controle reprodutivo por diversos agentes do Estado, em seus múltiplos níveis. Importante reter que o fenômeno da coerção contraceptiva não se deve à "maldade" de profissionais de saúde, mas obedece a um esquema mais amplo relativo à forma como políticas públicas são desenhadas, como programas e serviços de saúde são organizados, muitas vezes por meio de incentivos fiscais, aportes de recursos internacionais, dentre outras formas de apoio, que terminam por subordinar a autonomia reprodutiva como um princípio basilar destes programas.

Travestido com a linguagem dos direitos, este é um jogo que não apenas amplia as desigualdades sociais entre as mulheres, mas reforça dimensões racistas e estigmatizantes, ao também ignorar a imprescindibilidade do debate sobre justiça reprodutiva.

\section{Colaboradores}

ER Brandão concebeu o estudo, coletou os dados, analisou e redigiu o artigo. CS Cabral participou da discussão dos dados e redação do manuscrito.

\section{Agradecimentos}

Ao Conselho Nacional de Desenvolvimento Científico e Tecnológico (CNPq). À Soraya Fleischer, por texto precioso. 


\section{Referências}

1. Cabral CS, Brandão ER. Gravidez na adolescência, iniciação sexual e gênero: perspectivas em disputa. Cad Saude Publica 2020; 36(8):e00029420.

2. Federação Brasileira das Associações de Ginecologia e Obstetrícia (FEBRASGO). Posicionamento da FEBRASGO sobre a campanha de prevenção da gravidez na adolescência "Adolescência primeiro, gravidez depois" [Internet]. 2020 [acessado 2019 maio 15]. Disponível em: https://www.febrasgo.org.br/pt/noticias/ item/939-posicionamento-da-febrasgo-sobre-a-campanha-de-prevencao-da-gravidez-na-adolescencia-adolescencia-primeiro-gravidez-depois.

3. Pitanguy J, Barroso C. Sexo e gênero, a fabricação do perigo [Internet]. Folha de São Paulo; 2020 [acessado 2019 maio 15]. Disponível em: https://wwwl.folha. uol.com.br/opiniao/2020/02/sexo-e-genero-a-fabricacao-do-perigo.shtml.

4. Brasil. Ministério da Saúde (MS). Secretaria de Atenção em Saúde. Departamento de Ações Programáticas Estratégicas. Cuidando de Adolescentes: orientações básicas para a saúde sexual e a saúde reprodutiva. Brasília: MS; 2015.

5. Brasil. Ministério da Saúde (MS). Secretaria de Atenção à Saúde. Departamento de Ações Programáticas Estratégicas. Marco teórico e referencial: saúde sexual e saúde reprodutiva de adolescentes e jovens. Brasília: MS; 2006.

6. Ross L. What Is Reproductive Justice? In: Reproductive Justice Briefing Book. s/d. p. 4-5.

7. Vigoya MV. La interseccionalidad: una aproximación situada a la dominación. Debate Feminista 2016; 52:117.

8. Crenshaw K. Documento para o encontro de especialistas em aspectos da discriminação racial relativos ao gênero. Rev Estud Fem 2002; 10(1):171-188.

9. Price K. What is Reproductive Justice? How Women of Color Activists Are Redefining the Pro-Choice Paradigm. Meridians Fem Race Transnat 2010; 10(2):4265 .

10. Luna Z, Luker K. Reproductive Justice. Annu Rev Law Soc Sci 2013; 9:327-352.

11. Leal MC, Gama SGN, Pereira APE, Pacheco VE, Carmo CN, Santos RV. A cor da dor: iniquidades raciais na atenção pré-natal e ao parto no Brasil. Cad Saúde Pública 2017; 33(Supl. 1):e00078816.

12. Goes EF, Menezes GMS, Almeida MCC, Araújo TVB, Alves SV, Alves MTSSB, Aquino EML. Vulnerabilidade racial e barreiras individuais de mulheres em busca do primeiro atendimento pós-aborto. Cad Saude Publica 2020; 36(Supl. 1):e00189618.

13. Faya-Robles A. Da gravidez de "risco" às "maternidades de risco": biopolítica e regulações sanitárias nas experiências de mulheres de camadas populares de Recife. Physis 2015; 25(1):139-169.

14. Fernandes C. Figuras do constrangimento: as instituições de Estado e as políticas de acusação sexual. Mana 2019; 25(2):365-390.

15. Sarmento C. Mulheres em situação de rua e tecnologias de governo. In: Mendes N, Merhy E, Silveira P, organizadores. Extermínio dos excluídos. Porto Alegre: Rede Unida; 2019. p.89-102.
16. Carneiro S. A construção do outro como não-ser como fundamento do ser [tese]. São Paulo: Universidade de São Paulo; 2005.

17. Werneck J. O belo ou o puro? Racismo, eugenia e novas (bio)tecnologias. In: Rotania AA, Werneck J, organizadoras. Sob o signo das Bios: vozes críticas da sociedade civil. Rio de Janeiro: E-papers Serviços Editoriais; 2004.

18. Clarke A, Shim J. Medicalization and biomedicalization revisited: technoscience and transformations of health, illness and American medicine. In: Pescosolido BA, Martin JK, Mcleod JD, Rogers A, editors. Handbook of the sociology of health, illness, and healing. New York, Dordrecht, Heidelberg, London: Springer; 2011.p.173-199.

19. Valdez N, Deomampo D. Centering Race and Racism in Reproduction. Med Anthropol 2019; 38(7):551-559.

20. Rapp R. Race \& Reproduction: An Enduring Conversation. Med Anthropol 2019; 38(8):725-732.

21. Pimentel ACL, Jannotti CB, Gaudenzi P, Teixeira LAS A breve vida do Norplant ${ }^{\circledR}$ no Brasil: controvérsias e reagregações entre ciência, sociedade e Estado. Cien Saude Colet 2017; 22(1):43-52.

22. Manica D, Nucci M. Sob a pele: implantes subcutâneos, hormônios e gênero. Horiz Antropol 2017 23(47):93-129.

23. Federação Brasileira das Associações de Ginecologia e Obstetrícia (FEBRASGO). Contracepção reversível de longa ação. São Paulo: FEBRASGO; 2016.

24. Ponce de Leon RG, Ewerling F, Serruya SJ, Silveira MF, Sanhueza A, Moazzam A, Becerra-Posada F, Coll CVN, Hellwig F, Victora CG, Barros AJD. Contraceptive use in Latin America and the Caribbean with a focus on long-acting reversible contraceptives: prevalence and inequalities in 23 countries. Lancet Glob Health 2019; 7:e227-e235.

25. Gubrium AC, Mann ES, Borrero S, Dehlendorf C, Fields J, Geronimus AT, Gómez AM, Harris LH, Higgins JA, Kimport K, Luker K, Luna Z, Mamo L, Roberts D, Romero D, Sisson G. Realizing Reproductive Health Equity Needs More Than Long-Acting Reversible Contraception (LARC). Am J Public Health 2016; 106(1):18-19.

26. Senderowicz L. "I was obligated to accept": A qualitative exploration of contraceptive coercion. Soc Sci Med 2019; 239:112531.

27. Diário do Nordeste. CE éo $1^{\circ}$ do Brasil a usar implante contraceptivo [Internet]. 2010 [acessado 2019 nov 18]. Disponível em: https://diariodonordeste.verdesmares.com.br/editorias/metro/ce-e-o-1-do-brasil-a-usar -implante-contraceptivo-1.536907.

28. Diário do Nordeste. Método contraceptivo para mulheres em risco [Internet]. 2016 [acessado 2019 nov 18]. Disponível em: https://diariodonordeste.verdesmares.com.br/editorias/metro/metodo-contraceptivo -para-mulheres-em-risco-1.1588729.

29. Governo do Estado do Ceará. Mulheres em situação de risco terão alternativa de implante anticoncepcional [Internet]. 2016 [acessado 2019 nov 18]. Disponível em: https://www.ceara.gov.br/2016/07/19/mulheres -em-situacao-de-risco-terao-alternativa-de-implante -anticoncepcional/ 
30. Bezerra P. Projeto de Lei no 467 , de 09 setembro de 2015. Dispõe sobre política de proteção às mulheres em situação de vulnerabilidade, pela rede pública de saúde com a utilização do contraceptivo reversível de longa duração de etonogestrel, e dá outras providências. Câmara Municipal de São Paulo 2015; 09 set.

31. São Paulo. Lei no 16.806, de 19 de janeiro de 2018. Dispõe sobre política de proteção às mulheres em situação de vulnerabilidade pela Rede Pública de Saúde, com a utilização do Contraceptivo Reversível de Longa Duração de Etonogestrel, e dá outras providências. Diário Oficial São Paulo 2018; 20 jan.

32. Câmara Municipal de São Paulo. Agora é lei: contraceptivo de longa duração será disponibilizado na rede pública [Internet]. 2018 [acessado 2019 nov 18]. Disponível em: http://www.saopaulo.sp.leg.br/blog/agora-e-lei-contraceptivo-de-longa-duracao-sera-disponibilizado-na-rede-publica/.

33. Prefeitura de São Paulo. Rede Municipal adquire implantes contraceptivos de longa duração para ampliar gama do Planejamento Reprodutivo [Internet]. 2016 [acessado 2019 nov 18]. Disponível em: https://www. prefeitura.sp.gov.br/cidade/secretarias/saude/noticias/?p=211815.

34. São Paulo. Decreto no 58.693, de 2 de abril de 2019. Regulamenta a Lei no 16.806, de 19 de janeiro de 2018, que dispõe sobre política de proteção às mulheres em situação de vulnerabilidade pela Rede Pública de Saúde, com a utilização do Contraceptivo Reversível de Longa Duração de Etonogestrel. Diário Oficial São Paulo 2019; 02 abr.

35. Ministério Público do Estado do Rio Grande do Sul. MP assina termo de cooperação para garantir acesso das adolescentes acolhidas às politicas de saúde da mulher [Internet]. 2018 [acessado 2019 nov 18]. Disponível em: https://www.mprs.mp.br/noticias/infancia/46995/.

36. Ministério Público do Estado do Rio Grande do Sul. Promotoria de Justiça da Infância e da Juventude. Termo de Cooperação [Internet]. Porto Alegre; 2018 [acessado 2019 nov 18]. Disponível em: https://apublica.org/wp-content/uploads/2019/03/termo-de-cooperacao.pdf.

37. Conselho Municipal de Saúde de Porto Alegre. Nota Pública Conjunta [Internet]. Porto Alegre; 2018 [acessado 2019 nov 18]. Disponível em: http://www2.portoalegre.rs.gov.br/cms/default.php?reg=217\&p_se$\mathrm{caO}=8$.

38. Anjo AB. Quem decide sobre o corpo das meninas? [Internet]. Agência Pública; 2019 [acessado 2019 nov 18]. Disponível em: https://apublica.org/2019/03/ quem-decide-sobre-o-corpo-das-meninas/.
39. Tribunal Regional Federal da $4^{\text {a }}$ Região. TRF4 suspende aplicação de contraceptivo em adolescentes acoIhidas pela prefeitura de Porto Alegre [Internet]. 2019 [acessado 2019 nov 18]. Disponível em: https://www. trf4.jus.br/trf4/controlador.php?acao=noticia_visualizar\&id_noticia $=14345$.

40. Negrão T. Uma Ação Sinérgica por Direitos Reprodutivos: uma história sem fim. Saude Soc 2012; 21(Supl. 1):164-176.

41. Rede Nacional Feminista de Saúde, Direitos Sexuais e Direitos Reprodutivos. Regional do Rio Grande do Sul e outras. Implantes hormonais em jovens: a volta do controle da natalidade, uma violação aos direitos humanos [Internet]. Porto Alegre; 2006 [acessado 2019 nov 18]. Disponível em: https://www.ufrgs.br/nph/ ong/? $\mathrm{p}=701$.

42. Brandão ER. Métodos contraceptivos reversíveis de longa duração no Sistema Único de Saúde: o debate sobre a (in)disciplina da mulher. Cien Saude Colet 2019; 24(3):875-879.

43. Caetano AJ. Esterilização cirúrgica feminina no Brasil, 2000 a 2006: aderência à lei de planejamento familiar e demanda frustrada. Rev Bras Estud Popul 2014; 31(2):309-331.

44. Cavenaghi S, Alves JED. The everlasting outmoded contraceptive method mix in Brazil and its legacy. Rev Bras Estud Popul 2019; 36:e103.

45. Cabral CS. Contraception et stérilisation des jeunes femmes au Brésil. Autrepart 2014; 70:165-183.

46. Long-Acting Reversible Contraception (LARC). Statement of Principles. Atlanta: SisterSong Women of Color Reproductive Health Collective \& National Women Health Network; 2016.

Artigo apresentado em 29/04/2020

Aprovado em 12/04/2021

Versão final apresentada em 14/04/2021

Editores-chefes: Romeu Gomes, Antônio Augusto Moura da Silva 